\title{
Percentual de gordura corporal em idosos: comparação entre os métodos de estimativa pela área adiposa do braço, pela dobra cutânea tricipital e por bioimpedância tetrapolar
}

\author{
Percentage of body fat among elderly: comparison between the methods of \\ estimation by the mid-arm adipose area, tricipital skinfold thickness and \\ tetrapolar bioimpedance analysis \\ Renata Santos Pereira Machado ${ }^{1}$ \\ Maria Auxiliadora Santa Cruz Coelho² \\ Karla Santa Cruz Coelho ${ }^{3}$
}

Resumo

Objetivo: Comparar e avaliar o grau de concordância dos percentuais de gordura corporal em idosos por três diferentes métodos: pela área adiposa do braço (AAB), pela prega cutânea tricipital (PCT) e por bioimpedância (BIA). Métodos: Realizouse pesquisa quantitativa, descritiva, de corte transversal, com 395 idosos residentes em abrigos conveniados com o município do Rio de Janeiro - RJ. As variáveis utilizadas foram: medidas de massa corporal, estatura, circunferência da cintura, circunferência do braço, prega cutânea tricipital, Índice de Massa Corporal, circunferência muscular do braço, área muscular do braço, área do braço e a equação de Siri. Para associação das variáveis, utilizou-se o Coeficiente de Correlação de Pearson (r), $\alpha=0,05$, e para concordância, o Coeficiente de Correlação Intraclasse (CCI), com limite de 0,75. Resultados: Todos os coeficientes de correlação intraclasse (CCI) entre as variáveis foram estatisticamente significantes. Comparando os três métodos, o CCI foi de 0,855 , com Intervalo do Confiança (IC) de $(0,826-0,881)$. Todos os métodos também apresentaram elevada correlação com as variáveis antropométricas isoladamente, sendo maior a do percentual de gordura por bioimpedância com o IMC, $r=0,935 \mathrm{p}=0,000$. As mulheres apresentaram maior percentual de gordura pelos três métodos, com $\mathrm{p}<001$. Conclusão: Todos percentuais de gordura apresentaram associação estatisticamente significante com as variáveis antropométricas, $\mathrm{o} \% \mathrm{G}_{\mathrm{BIA}}$ apresentou melhor correlação com o IMC, que reflete a massa corporal total e com a CC, que reflete o depósito abdominal. As mulheres tiveram maior percentual de gordura corporal. $\mathrm{O} \% \mathrm{G}_{\mathrm{AAB}}$ e o \% $\mathrm{G}_{\mathrm{SIRI}}$ tiveram boa concordância entre eles, sendo que ambos refletem medidas nos membros superiores. $O$ método utilizado influi na estimativa do percentual de gordura a partir dos membros superiores. A AAB sobreestima o percentual de gordura com relação à Equação de Siri e BIA, e seu uso como estimador de gordura global deve ser mais bem estudado.

\footnotetext{
Universidade Federal Rural do Rio de Janeiro. Instituto Multidisciplinar. Rio de Janeiro, RJ, Brasil.

2 Universidade Federal do Rio de Janeiro. Instituto de Nutrição Josué de Castro. Departamento de Nutrição Social Aplicada. Rio de Janeiro, RJ, Brasil.

3 Agência Nacional de Saúde Suplementar. Rio de Janeiro, RJ, Brasil.
}

Palavras-chave: Idoso. Distribuição da Gordura Corporal. Antropometria. Composição Corporal. Avaliação Nutricional. Adiposidade. Pregas Cutâneas. Estudo Comparativo. Análise Quantitativa. Estudos Transversais. Rio de Janeiro, RJ. Percentual de Gordura 


\section{Abstract}

Objective: Compare and evaluate the agreement degree of the body fat percentages of $(\% \mathrm{BF})$ estimated by mid-arm adipose area (\%BF-ARM), the Siri triceps skinfold equation (\%BF-SIRI) and the tetrapolar bioimpedance analysis (\%BF-BIA) in the elderly. Methods: A cross-sectional study, with 395 elderly residents in shelters of Rio de Janeiro, Brazil. Anthropometric measures were weight (W), height $(\mathrm{H})$, waist circumference (WC) mid-arm circumference (ARM) and triceps skinfold (TS).It was calculated the Body Mass Index (BMI). For \%BF were used the equation of Siri, apparatus RJL tetrapolar to BIA, and calculated \%BF-ARM. P-value was 0.05. For correlation it was used Pearson's Coefficient ( $\mathrm{r}$ ), and for agreement, the Intraclass Correlation Coefficient (ICC), with limit of 0.75. Results: All Intra-class Correlation Coefficients were statistically significant. Comparing three methods, the ICC was 0,855, with 0,826-0,881 Confidence Interval (CI). All methods also presented high correlation with the anthropometric measures. The highest correlation was between body mass index and biomimpedance, $r=0,935 p=0,000$. Women presented the highest body fat percentual, for the three methods, with $\mathrm{p}<0.001$. Conclusion: All \%BF showed statistically significant association with the anthropometric variables, the \%BF-BIA had better correlation with BMI that reflects the global body mass and with the WC that reflects the abdominal fat. Women had a higher percentage of body fat. The \%BF-ARM and \%BF-SIRI had good agreement between them, both reflecting measures in the upper limbs. The method used influences the estimate of percentage of body fat of the upper limbs. The mid-arm adipose area overestimated the percentage of fat in relation to the equation of Siri and BIA, and its use as an estimator of fat overall should be better studied.
Key words: Aged. Body Fat Distribution.

Anthropometry. Body Composition. Nutrition Assessment. Adiposity. Skinfold Thickness. Comparative Study. Quantitative Analysis. Cross-Sectional Studies. Rio de Janeiro city. Elderly. Percentage of Body fat.

\section{INTRODUÇÃO}

A população idosa vem aumentando no Brasil, representando, em 2002, um total de mais de 15 milhões de pessoas. Para o ano de 2050, a expectativa no Brasil, bem como em todo o mundo, é de que existirão mais idosos que crianças abaixo de 15 anos. ${ }^{1,2}$

O envelhecimento populacional é um fenômeno mundial, e tem aumentado a necessidade do estudo da adequação de padrões desenvolvidos para populações mais jovens e criação de novos padrões para os idosos, uma vez que, com o passar do tempo, o organismo sofre mudanças de ordem biológica e fisiológica, que vão influenciar no estado nutricional do idoso, podendo afetar sua acurácia e precisão. ${ }^{1-4}$

O estado nutricional influi na manutenção da saúde e na morbi-mortalidade associada a múltiplos processos crônicos, ${ }^{5.7}$ sendo de grande relevância na manutenção da qualidade de vida dos idosos. ${ }^{8-10}$
Os indicadores antropométricos são ferramentas úteis na avaliação do estado nutricional, sendo largamente utilizados em pesquisas epidemiológicas, por serem de mais fácil obtenção, de baixo custo e permitirem resultados mais rápidos para estudos populacionais, sendo também relevantes os indicadores da composição corporal. O indicador antropométrico mais utilizado é o Índice de Massa Corporal (IMC), que apesar de ser de grande utilidade e de larga aplicação em estudos epidemiológicos, ele não permite determinar a composição corporal. ${ }^{11}$

A estimação da gordura corporal pode ser feita através de diversos métodos, sendo alguns de custo mais elevado e mais difícil acesso, como o de hidrodensitometria, absormetria de raios $\mathrm{X}$ de dupla energia (DEXA) e ressonância magnética nuclear, e outros mais acessíveis, como a bioimpedância e equações que aplicam medidas antropométricas, tais como a circunferência do braço e a prega cutânea tricipital. ${ }^{10,11}$ 
O objetivo deste trabalho é avaliar o grau de concordância entre os percentuais de gordura corporal estimados por: biompedância tetrapolar, área adiposa do braço e prega cutânea tricipital aplicada na Equação de Siri em idosos.

\section{METODOLOGIA}

Este estudo faz parte de uma pesquisa maior intitulada "Perfil de saúde e nutrição de idosos residentes em abrigos do município do Rio de Janeiro", do Instituto de Nutrição da UFRJ, de desenho transversal, no qual foram analisados 395 idosos residentes em instituições asilares conveniadas com o município do Rio de Janeiro - RJ no ano de 2000.

Foram realizadas medidas antropométricas de peso $(\mathrm{P})$, estatura $(\mathrm{E})$, circunferência da cintura (CC) circunferência do braço (CB) e prega cutânea tricipital (PCT), e calculados o Indice de Massa Corporal (IMC), a circunferência muscular do braço (CMB), a área muscular do braço (AMB), a área do braço $(\mathrm{AB})$ e a área adiposa do braço (AAB), segundo o critério de Frisancho. ${ }^{12}$ Todas as medidas antropométricas foram aferidas pelo lado dominante em duplicata, e a PCT em triplicata, e calculada a média destes valores.

Para aferição da massa corporal, utilizou-se balança Eletronic Digital Kratos do tipo portátil eletrônica, com capacidade máxima de $150 \mathrm{~kg}$. No momento de aferição da massa corporal, os idosos foram solicitados a retirar sapatos, vestes e relógios, pulseiras, chapéu, boné, cinto, xale e demais acessórios que acarretassem aumento de massa corporal. A massa corporal considerada foi aquela no $0,1 \mathrm{~kg}$ mais próximo. ${ }^{13}$

A estatura foi medida em metros, utilizando antropômetro vertical para adultos, da marca Leicester Height Measure. Os idosos tiveram suas medidas aferidas estando descalços, em posição ortostática, com as superfícies posteriores de calcanhar, cintura pélvica, cintura escapular e região occipital o mais próximo possível do antropômetro, com a cabeça orientada segundo o plano de Frankfurt, paralela ao solo. A medida foi feita com o cursor em ângulo de $90^{\circ} \mathrm{em}$ relação à escala. A leitura foi feita no $0,5 \mathrm{~cm}$ mais próximo, quando a haste horizontal, perpendicular à barra vertical da escala de estatura, encostasse no vértex da cabeça. ${ }^{13}$

A medida de dobra cutânea triciptal foi determinada paralelamente ao eixo longitudinal do braço direito, na face posterior, sendo seu ponto exato de medição a distância média entre a borda súpero-lateral do acrômio e o olécrano. ${ }^{14}$ O idoso, com o braço estendido ao longo do corpo, com a palma da mão voltada para frente, tinha sua dobra cutânea medida com um adipômetro de marca Lange.

O ponto de verificação da medida da circunferência do braço foi obtido estando o cotovelo do idoso flexionado a 90 graus e a palma da mão voltada para o tórax. O local da aferição foi determinado no ponto médio do braço, localizado entre o processo acromial e o olécrano, onde foi feita uma marcação com lápis dermográfico. O idoso, com o braço estendido ao longo do corpo, com a palma da mão voltada para frente, tinha sua circunferência do braço medida através de uma fita métrica flexível e inelástica, com escala em milímetro, da marca Sanny. $\mathrm{O}$ avaliador realizava a medida no ponto médio do braço, sem comprimir os tecidos. ${ }^{14}$

A medida da CB foi usada, associada à dobra cutânea tricipital, para calcular a área muscular e adiposa do braço, através da equação de Frisancho. ${ }^{12}$

Para medida da circunferência da cintura, o examinador ficou de frente para o examinando e colocou a fita métrica horizontalmente ao redor da cintura natural ou na menor curvatura localizada entre as costelas e a crista ilíaca, preconizada pela WHO (1995). ${ }^{14}$

Para estimativa do percentual de gordura pela PCT, utilizou-se a equação de Siri. O percentual de gordura estimado pela biompedância tetrapolar foi feito com o uso do aparelho RJL, que requer, para o cálculo percentual de gordura, informações sobre sexo, idade, estatura, massa corporal e 
atividade física. O IMC foi calculado pela equação peso dividido pelo quadrado da altura. As medidas foram aferidas conforme os critérios descritos pela Organização Mundial de Saúde. ${ }^{14,15}$ As equações utilizadas foram:

Circunferência Muscular do Braço (CMB):

$(\mathrm{CMB})=\mathrm{CB}-(3,14 * \mathrm{PCT})$

Área muscular do braço (AMB):

$(\mathrm{AMB})=(\mathrm{CMB}) 2 /(4 * 3,14)$

Área adiposa do braço (AAB):

$(\mathrm{AAB})=\mathrm{PCT}$ em cm $*(\mathrm{CB} / 2)-((3,14 *(\mathrm{PCT}$ em cm)2) / 4)

Percentual de gordura estimado pela $\mathrm{AAB}$ (\%GAAB):

$\% \mathrm{GAAB}=(\mathrm{AAB} \times 100) /(\mathrm{AAB}+\mathrm{AMB})$

Percentual de gordura pela PCT aplicada na equação de Siri (\%GSIRI):

$\%$ GSIRI $=((4,95 / D)-4,5) \times 100, c o m$

$\mathrm{D}=\mathrm{C}-\mathrm{M} \times \log 10 \mathrm{PT}$

Os coeficientes " $C$ " e " $M$ " são obtidos de tabela específicas, onde se especificam os valores em função do sexo e idade, ainda que de forma genérica seja possível adotar os seguintes valores para "C" e "M": C (homens: 1,1143; mulheres: 1,1278) e M (homens: 0,618; mulheres: 0,0775). ${ }^{16}$ A equação de Siri é reconhecida pela Sociedad Española para el Estúdio de la Obesidad (SEEDO) como técnica padrão para avaliar o percentual de gordura corporal. ${ }^{16,17}$

Para análise estatística, foram calculadas medidas de estatística descritiva, incluindo média, desvio padrão e valores mínimo e máximo para as variáveis contínuas, e o teste ANOVA para diferença entre médias. Para correlação, utilizouse o Coeficiente de Correlação de Pearson (r), e para concordância, o Coeficiente de Correlação Intraclasse (CCI), com limite de 0,75..$^{18,19}$ Definiuse para este estudo o nível de significância de $\alpha=0,05$. Os dados foram analisados com o pacote estatístico SPSS versão 11.0.

O protocolo de pesquisa foi aprovado pela Comissão de Ética do Hospital Universitário Clementino Fraga Filho, da Universidade Federal do Rio de Janeiro.

\section{RESULTADOS}

Foram avaliados 395 idosos residentes em instituições asilares no município do Rio de Janeiro no ano de 2000. A prevalência do sexo foi de $41,3 \%$ (163) de homens e $58,7 \%$ (232) de mulheres.

Na tabela 1, estão apresentados os valores médios, com desvio padrão das variáveis antropométricas e de composição corporal utilizados. A idade média foi de 74,8 anos (DP=9,8), o IMC médio foi de 24,7 $(\mathrm{DP}=5,5)$ dentro da classificação de eutrofia segundo os critérios da Organização Mundial de Saúde (OMS). ${ }^{15}$ Já as médias dos percentuais de gordura estimadosforam de 16,8 $(\mathrm{DP}=10,4)$ pelabioimpedância (BIA), 32,8 ( $\mathrm{DP}=12,7)$ pela área adiposa do braço $(\mathrm{AAB})$ e 26,6 $(\mathrm{DP}=8,7)$ pela Equação de Siri. Estratificando por sexo, observa-se que homens possuem maior estatura e peso, e menor IMC. Já a média da PCT das mulheres é quase o dobro da dos homens. O percentual de gordura teve a média maior para as mulheres nos três métodos utilizados. 
Tabela 1 - Características gerais, medidas antropométricas e composição corporal de idosos de acordo com o sexo. Rio de Janeiro, RJ

\begin{tabular}{lllll}
\hline Variáveis & $\begin{array}{c}\text { Total } \\
\text { média } \pm \mathrm{DP} \\
(\mathrm{n})\end{array}$ & $\begin{array}{l}\text { Homens } \\
\text { Média } \pm \mathrm{DP} \\
(\mathrm{n})\end{array}$ & $\begin{array}{l}\text { Mulheres } \\
\text { média } \pm \mathrm{DP} \\
(\mathrm{n})\end{array}$ & p-valor \\
\hline Idade (anos) & $74,8 \pm 9,8$ & $73 \pm 8,7$ & $76 \pm 10,2$ & \\
& $(395)$ & $(163)$ & $(232)$ & 0,030 \\
Altura (cm) & $155,5 \pm 10,5$ & $163,2 \pm 7,7$ & $150,1 \pm 8,7$ & 0,000 \\
& $(369)$ & $(152)$ & $(217)$ & \\
Peso $(\mathrm{Kg})$ & $59,6 \pm 14,0$ & $62,0 \pm 12,6$ & $57,88 \pm 14,6$ & 0,005 \\
& $(366)$ & $(150)$ & $(216)$ & \\
IMC (Kg/m2) & $24,7 \pm 5,5$ & $23,24 \pm 4,1$ & $25,70 \pm 6,2$ & 0,000 \\
& $(366)$ & $(150)$ & $(216)$ & \\
Circunferência do & $27,7 \pm 4,5$ & $27,30 \pm 3,4$ & $27,95 \pm 5,0$ & 0,188 \\
Braço (cm) & $(336)$ & $(136)$ & $(200)$ & \\
Circunferência da & $88 \pm 13,9$ & $88,99 \pm 12,7$ & $87,23 \pm 14,2$ & 0,208 \\
cintura (cm) & $(335)$ & $(150)$ & $(199)$ & \\
Prega Cutânea & $16,8 \pm 8,8$ & $11,71 \pm 5,3$ & $20,20 \pm 9,0$ & 0,000 \\
Tricipital (mm) & $(332)$ & $(134)$ & $(198)$ & \\
Percentual de & $24,7 \pm 10,4$ & $23,28 \pm 4,1$ & $25,70 \pm 6,5$ & 0,000 \\
gordura BIA & $(347)$ & $(146)$ & $(213)$ & \\
Percentual de & $32,8 \pm 12,7$ & $24,31 \pm 8,7$ & $38,48 \pm 11,7$ & 0,000 \\
Gordura AAB & $(332)$ & $(134)$ & $(198)$ & \\
Percentual de & $26,6 \pm 8,7$ & $20,98 \pm 5,7$ & $30,39 \pm 8,2$ & 0,000 \\
Gordura Siri & $(332)$ & $(134)$ & $(198)$ & \\
\hline
\end{tabular}

IMC = Índice de Massa Corporal; BIA = bioimpedância; $\mathrm{AAB}=$ área adiposa do braço

A correlação através do Coeficiente de Correlação de Pearson (r) está demonstrada na tabela 2. Todas as associações apresentaram correlação positiva, com elevada significância estatística, com p-valor menor que 0,001. O percentual de gordura estimado pela bioimpedância (\%GBIA) teve boa correlação com o percentual de gordura estimado pela prega cutânea tricipital através da equação de Siri (\%GSiri), com $r=0,665$ $(\mathrm{p}<0,0001)$, e também com o estimado pela área adiposa do braço (\%GAAB), com $r=0,550$ ( $\mathrm{p}<0,0001)$. Já o \%GAAB apresentou correlação mais forte para o \%GSiri, com $r=0,959$ $(\mathrm{p}<0,0001)$. As demais variáveis antropométricas também apresentaram boa correlação entre elas, com significância estatística. Separando-se por sexo, os homens apresentaram maior correlação do \%GBIA com as variáveis IMC e CB, e as mulheres maior correlação do \%GAAB e \%GSiri com as variáveis IMC, CC e CB. 
Tabela 2 - Grau de associação entre as variáveis estudadas em idosos de acordo com o sexo. Rio de Janeiro, RJ

\begin{tabular}{|c|c|c|c|c|c|c|c|}
\hline Variáveis & IMC & CC & CB & PCT & \%GBIA & $\% G A A B$ & \%GSIRI \\
\hline & $r *$ & & & & & & \\
\hline \multicolumn{8}{|l|}{ TOTAL } \\
\hline IMC & 1 & 0,741 & 0,775 & 0,706 & 0,935 & 0,577 & 0,674 \\
\hline CC & 0,741 & 1 & 0,670 & 0,495 & 0,736 & 0,366 & 0,456 \\
\hline $\mathrm{CB}$ & 0,779 & 0,670 & 1 & 0,733 & 0,789 & 0,513 & 0,694 \\
\hline PCT & 0,706 & 0,495 & 0,733 & 1 & 0,686 & 0,942 & 0,694 \\
\hline$\%$ GBIA & 0,935 & 0,736 & 0,789 & 0,686 & 1 & 0,550 & 0,665 \\
\hline$\% G A A B$ & 0,577 & 0,366 & 0,513 & 0,942 & 0,550 & 1 & 0,959 \\
\hline \%GSIRI & 0,674 & 0,456 & 0,694 & 0,966 & 0,665 & 0,959 & 1 \\
\hline \multicolumn{8}{|l|}{ HOMENS } \\
\hline IMC & 1 & 0,763 & 0,796 & 0,620 & 0,972 & 0,478 & 0,630 \\
\hline CC & 0,763 & 1 & 0,677 & 0,504 & 0,771 & 0,387 & 0,518 \\
\hline $\mathrm{CB}$ & 0,796 & 0,677 & 1 & 0,671 & 0,804 & 0,493 & 0,695 \\
\hline PCT & 0,620 & 0,504 & 0,671 & 1 & 0,647 & 0,968 & 0,976 \\
\hline$\%$ GBIA & 0,972 & 0,771 & 0,804 & 0,647 & 1 & 0,509 & 0,656 \\
\hline$\%$ GAAB & 0,478 & 0,380 & 0,493 & 0,968 & 0,509 & 1 & 0,958 \\
\hline \%GSIRI & 0,630 & 0,511 & 0,695 & 0,976 & 0,656 & 0,958 & 1 \\
\hline \multicolumn{8}{|c|}{ MULHERES } \\
\hline IMC & 1 & 0,780 & 0,776 & 0,742 & 0,921 & 0,616 & 0,715 \\
\hline $\mathrm{CC}$ & 0,780 & 1 & 0,691 & 0,664 & 0,771 & 0,548 & 0,636 \\
\hline $\mathrm{CB}$ & 0,776 & 0,691 & 1 & 0,834 & 0,786 & 0,598 & 0,807 \\
\hline PCT & 0,742 & 0,664 & 0,834 & 1 & 0,710 & 0,919 & 0,955 \\
\hline$\%$ GBIA & 0,921 & 0,771 & 0,786 & 0,710 & 1 & 0,571 & 0,684 \\
\hline$\%$ GAAB & 0,616 & 0,548 & 0,598 & 0,919 & 0,571 & 1 & 0,938 \\
\hline \%GSIRI & 0,715 & 0,636 & 0,807 & 0,955 & 0,684 & 0,938 & 1 \\
\hline
\end{tabular}

$\mathrm{r}=$ Coeficiente de Correlação de Pearson; IMC = Índice de Massa Corporal; $\mathrm{CC}=$ Circunferência da Cintura; $\mathrm{CB}=\mathrm{Circunfe-}$ rência do braço; \%GBIA = Percentual de gordura pela Bioimpedância; \%GAAB = Percentual de gordura pela área adiposa do braço; \%GSIRI = Percentual de gordura pela prega cutânea tricipital aplicada na Equação de Siri

*Todas as variáveis apresentaram correlação com p-valor $<0,01$.

A tabela 3 descreve a concordância entra as diferentes estimativas de percentual de gordura através do Coeficiente de Correlação Intraclasse (CCI) com os respectivos Intervalos de Confiança (IC) de 95\%. Observa-se que a maioria das associações apresenta concordância acima do limite de 0,75 , e todos apresentaram significância estatística, observando os intervalos de confiança. A concordância entre os três percentuais estimados foi elevada, de 0,855 (IC =0,826-0,881), sendo o maior CCI entre \%GAAB e \%GSiri, de 0,944 (IC=0,930$0,955)$. Ao estratificar por sexo a concordância entre $\%$ GBIA e $\%$ GAAB permanece abaixo do ponto de corte de 0,75 para homens e mulheres. 
Tabela 3 - Grau de concordância entre os diferentes métodos de determinação de gordura corporal em idosos de acordo com o sexo. Rio de Janeiro, RJ

\begin{tabular}{lll}
\hline Variáveis & CCI & IC95\% \\
\hline \%GBIA + \%GAAB + \%GSIRI & Total & \\
\%GBIA + \%GAAB & 0,855 & $0,826-0,881$ \\
\%GBIA + \%GSIRI & 0,585 & $0,484-0,667$ \\
\%GAAB + \%GSIRI & 0,752 & $0,692-0,801$ \\
& 0,944 & $0,930-0,955$ \\
\%GBIA + \%GAAB + \%GSIRI & Homens & $0,793-0,887$ \\
\%GBIA + \%GAAB & 0,846 & $0,379-0,690$ \\
\%GBIA + \%GSIRI & 0,561 & $0,669-0,835$ \\
\%GAAB + \%GSIRI & 0,766 & $0,909-0,954$ \\
\%GBIA + \%GAAB + \%GSIRI & 0,935 & \\
\%GBIA + \%GAAB & Mulheres & $0,836-0,899$ \\
\%GBIA + \%GSIRI & 0,653 & $0,540-0,738$ \\
\%GAAB + \%GSIRI & 0,799 & $0,733-0,848$ \\
\hline
\end{tabular}

$\mathrm{CCI}=$ Coeficiente de Correlação Intraclasse; $\mathrm{IC}=$ Intervalo de Confiança; \%GBIA = Percentual de gordura pela bioimpedância; \%GAAB = Percentual de gordura pela área adiposa do braço; \%GSIRI = Percentual de gordura pela prega cutânea tricipital aplicada na Equação de Siri.

\section{DISCUSSÃO}

A avaliação da composição corporal dos idosos é muito importante, pois alterações na distribuição de gordura corporal podem estar associadas a desordens metabólicas e cardiovasculares. ${ }^{20}$

Embora tenham sido utilizados três métodos de estimativa indireta do percentual de gordura corporal dos idosos, as limitações de cada método não comprometem o objetivo do estudo.

O método de bioimpedância, baseado nas diferentes características condutoras da massa de gordura corporal e da massa livre de gordura devido ao seu diferente grau de hidratação, pode ser utilizado devido a sua rapidez, preço relativamente barato, precisão, simplicidade e facilidade de transporte e manuseio. Apesar de pequenas limitações ao seu uso, como o grau de hidratação e consumo de bebidas alcoólicas, o método vem sendo largamente citado pela literatura, com diversos estudos de validação e concordância. ${ }^{10,11,20,21,22}$
Com relação ao método de estimativa do percentual de gordura pela área adiposa do braço, o mesmo utiliza duas medidas antropométricas, a circunferência do braço e prega cutânea tricipital, existindo padrões de referência específicos para idosos propostos por Frisancho ${ }^{12}$ e referendados por outros autores. ${ }^{21-24}$

A equação de Siri utiliza a prega cutânea tricipital, cuja medida pode ser influenciada pela diminuição da elasticidade e hidratação da pele, relacionada ä idade, bem como pela redistribuição do tecido adiposo no idoso. Entretanto, seu uso é recomendado por estudiosos e pela Sociedad Española para el Estudio de la Obesidad. ${ }^{16,21,26-28}$

As medidas de prega cutânea são bastante usadas na estimativa da gordura corporal, por serem relativamente fáceis, de baixo custo e aplicáveis em estudos de campo. Apesar de divergências na literatura sobre o uso de dobras cutâneas em idosos, a mais utilizada é a dobra cutânea tricipital. ${ }^{21,23,24}$

Observa-se, ainda, que em todos os métodos utilizados não há referências específicas para 
indivíduos idosos brasileiros, tornando relevantes estudos com esta população.

Na tabela 1, comparando-se as médias de homens e mulheres, nota-se que as mulheres são mais idosas, possuem maior altura, menor peso, maior IMC e maior percentual de gordura pelos três métodos utilizados.

As médias do percentual de gordura no total foram diferentes entre os três métodos, conforme a tabela 1.

Observou-se elevada diferença entre as médias de PCT, o valor das mulheres correspondendo quase ao dobro do dos homens (tabela 1). Este achado já foi referido também em outros estudos com idosos. Meneses \& Marucci, ${ }^{29}$ em estudo com 483 idosos em Fortaleza - CE, encontraram uma média de PCT de $13 \mathrm{~mm}$ para homens e de 21,3 para mulheres, com $\mathrm{p}=0,000$. Outro estudo com idosos na região de São Paulo encontrou dados semelhantes, com PCT de 13,7 mm para homens e $25,2 \mathrm{~mm}$ para mulheres. ${ }^{30}$

Os resultados deste estudo indicam maior acúmulo de gordura subcutânea nas mulheres em relação aos homens, assim como foi encontrado em outros estudos no Brasil e no mundo. ${ }^{30-34}$

O valor médio da $\mathrm{CB}$ foi bem próximo entre os homens e as mulheres, sem diferença estatisticamente significante. Não foi possível observar diferença entre os sexos, provavelmente pelas características fisiológicas de cada um, com os homens apresentando maior massa muscular e as mulheres mais gordura, não sendo possível diferenciar ambos com esta medida isoladamente. ${ }^{35,36}$

Os três métodos apresentaram boa correlação com as medidas antropométricas de IMC, CC e CB. Todas as correlações foram estatisticamente significantes, sendo a melhor correlação do IMC com o percentual de gordura pela GBIA, seguida pela Equação de Siri e depois pela $A A B$, observando-se a mesma tendência para CC. Já a $\mathrm{CB}$ apresentou melhor correlação com a Equação de Siri, seguida pela BIA e pela AAB (tabela 2).
O percentual de gordura pela BIA tetrapolar apresentou maior correlação com as variáveis IMC e CB no sexo masculino. Por outro lado, as variáveis que sofrem maior influência do depósito de gordura abdominal e tricipital apresentaram maior correlação com o \%GBIA nas mulheres, assim como o \%GAAB e \%Siri com IMC, CC e CB.

Neste estudo, o CCI encontra-se na maioria os casos acima de 0,75 , indicando um grau de concordância muito bom (tabela 3).

Tendo em vista ser um estudo epidemiológico, não foi realizado nenhum dos métodos considerados pela literatura da área como padrão ouro, devido a problemas operacionais, como é o caso da pesagem hidrostática, tomografia computadorizada, entre outros. Este fato limita nossas observações acerca da metodologia mais apropriada para ser empregada na determinação da composição corporal no caso específico de idosos.

Os Coeficientes de Correlação Intraclasse entre os três diferentes métodos de estimativa do percentual de gordura foram bons, acima do ponto de corte de 0,75 , sendo semelhantes entre homens e mulheres. Houve uma exceção para o CCI entre $\%$ GBIA e \%GAAB, que foi abaixo do ponto de corte, tendência que se manteve na estratificação por sexo. Uma hipótese seria a do uso da $\mathrm{CB}$ na fórmula do \%GAAB, que reflete o depósito de gordura subcutânea nos membros superiores, havendo grande variação entre os sexos sem que seja possível diferenciá-los, enquanto o \%GBIA leva em consideração uma distribuição mais equitativa. Este dado sugere que a utilização da $\%$ GAAB como estimador de adiposidade global pode não ser válida.

Além disso, diversos autores descrevem a influência da utilização de um ou mais parâmetros antropométricos na equação utilizada para estimar o percentual de gordura corporal. ${ }^{21,37,38}$ Assim, para calcular o \%GSiri, só é necessário medir PCT, enquanto que para calcular \%GAAB é necessário medir PCT e CB. A presença deste segundo parâmetro na equação, e sua posição nela, possibilita uma variabilidade no resultado oferecido, já que mantido constante o valor da 
PCT, o valor da CB leva a um aumento maior da área muscular que da área adiposa, levando a um menor \%GAAB.

\section{CONCLUSÃO}

O estado nutricional contribui para o desenvolvimento de fatores que influenciam na morbi-mortalidade associada a múltiplos processos crônicos, de grande relevância na qualidade de vida dos idosos. Portanto, o estado nutricional traduz um processo dinâmico de relações entre fatores de ordem biológica, psíquica e social.

No que tange à avaliação nutricional, a medida mais utilizada é o IMC; no entanto, este indicador isolado não traz informações sobre a composição corporal. A associação com a avaliação de outros indicadores como as variáveis antropométricas, o $\% \mathrm{G}_{\mathrm{BIA}}{ }^{\% \mathrm{G}_{\mathrm{SIRI}}}$ e a BIA

\section{REFERÊNCIAS}

1. Instituto Brasileiro de Geografia e Estatística. Perfil dos Idosos responsáveis pelo domicílio no Brasil 2000. Estudos e Pesquisas Informação Demográfica e Socioeconômica 2002; 9.

2. Pereira RS, Curioni CC, Veras RP. Perfil demográfico da população idosa no Brasil e no Rio de Janeiro em 2002. Textos sobre Envelhecimento 2003; 6(1).

3. Brasil. Ministério da Saúde. Secretaria de Atenção à Saúde. Departamento de Atenção Básica. Envelhecimento e saúde da pessoa idosa. Brasília: Ministério da Saúde, 2006. 192 p. Cadernos de Atenção Básica,19. Série A. Normas e Manuais Técnicos.

4. Demographic Yearbook. Recent demographic developments in Europe Council of Europe Publishing 2004.

5. Esteban Pérez M, Fernández-Ballart J, SalasSalvadó J. Estado nutricional de la población anciana en función del régimen de institucionalización. Nutr Hosp 2000; 15: 105-13.

6. Ferrero López MI, Botella Trelis JJ. Alimentación y nutrición en la enfermedad de Alzheimer. Nutr Hosp 2000; 15: 280-90. demonstraram correlações significativas para avaliação do estado nutricional de idosos.

Neste estudo, todos os percentuais de gordura apresentaram associação estatisticamente significante com as variáveis antropométricas; o $\% \mathrm{G}_{\mathrm{BIA}}$ apresentou melhor correlação com o IMC, que reflete a massa corporal total e com a CC, que reflete o depósito abdominal. As mulheres tiveram maior percentual de gordura corporal. $\mathrm{O}$ $\% \mathrm{G}_{\mathrm{AAB}}$ e o \% $\mathrm{G}_{\text {SIRI }}$ tiveram boa concordância entre eles, sendo que ambos refletem medidas nos membros superiores. $\mathrm{O}$ método utilizado influi na estimativa do percentual de gordura a partir dos membros superiores. A AAB sobreestima o percentual de gordura com relação à Equação de Siri e BIA, e seu uso como estimador de gordura global deve ser mais bem estudado. A avaliação nutricional dos idosos associada a uma avaliação global são essenciais na prevenção e promoção de uma vida saudável ao envelhecer.
7. Kumar NB, Cantor A, Allen K, Cox CE. Android obesity at diagnosis and breast carcinoma survival: Evaluation of the effects of anthropometric variables at diagnosis, including body composition and body fat distribution and weight gain during lifespan, and survival from breast carcinoma. Cancer 2000; 88: 2751-7.

8. Mias C, Jurschik P, Massoni T, Sadurní M, Aguilà JJ, Solá R, Nuin C, Torres J. Evaluación Del estado nutriconal de los pacientes mayores atendidos em uma unidad de hospitalización a domicílio, Nutr Hosp 2003;18 (1):6-14.

9. Venegas Moreno E, Soto Moreno A, Pereira Cunill JL, García Peris P, León Sanz M, Pita Mercé AM, García-Luna PP. Pacientes en riesgo de desnutrición en asistencia primaria. Estudio sociosanitario. Nutr Hosp 2001; 16: 1-6.

10. Coelho MASC, Pereira RS, Coelho KSC. Antropometria e composição corporal em idosos. In: Frank AA, Soares EA, organizadores. Nutrição no envelhecer. 2 ed. Rio de Janeiro: Atheneu; 2002.

11. Coelho MASC, Amorim RB. Avaliação nutricional em Geriatria. In: Duarte ACG. 
Avaliação nutricional: aspectos clínicos e laboratoriais. São Paulo: Atheneu; 2007. Cap. 15. p.155-94.

12. Frisancho AR. New normams of upper limb fat and muscle areas for assessment of nutritional status. Am J Clin Nutr 1981; 34 (11):2540-5.

13. Gibson RS. Principles of nutrition assessment. New York: Oxford University Press; 1990. p. 691.

14. World Heath Organization. Report of the Expert Committee on Physical Status: the use and interpretation of anthropometry. Genova: WHO; 1995.

15. World Heath Organization. Obesity: preventing and managing the global epidemic. Report of a WHO Consultation. Genova: WHO; 2000. WHO Technical report series 894.

16. Sociedad Española para el Estudio de la Obesidad (SEEDO). Consenso español 1995 para la evaluación de la obesidad y para la realización de estudios epidemiológicos. Med Clin (Barc) 1996; 107: 782-7.

17. Siri WE. Body composition from fluid spaces and density: analysis of methods. In: Brozeck J, Henschel A, editores. Techniques for measuring body composition. Washington DC: National Academy of Sciences, Natural Resource Council; 1961.p. 223-44.

18. Griffin D, Gonzalez, R. Correlational analysis of dyad-level data in the exchangeable case. Psychol Bull 1995; 118: 430-9.

19. Shrout PE, Fleiss JL. Intraclass correlations: uses in assessing rater reliability. Psychol Bull 1979; 2: 420-8.

20. Barbosa AR,Santarém JM, Jacob Filho W, Meireles ES, Marucci MFN. Comparação da gordura corporal de mulheres idosas segundo antropometria, bioimpedância e DEXA. Arquivos Latinoamericanos de Nutrição 2001 mar; 51(1, supl 51).

21. Martín Moreno V, Gómez, Gandoy JBG, Antoranz MJ,Gómez de la Cámara A. Concordancia entre los porcentajes de grasa corporal estimados mediante el área ... Rev Esp Salud Pública 2003; 77(3).

22. Martín Moreno V, Gómez Gandoy JB, Antoranz González M J. Medición de la grasa corporal mediante impedancia bioeléctrica, pliegues cutáneos y ecuaciones a partir de medidas antropométricas. Análisis comparativo. Rev Esp Salud Pública 2001; 75: 221-36.

23. Alastrué A, Rull M, Camps I, Salvá JA. Nuevas normas y consejos en la valoración de los parámetros antropométricos en nuestra población: índice adiposo muscular, índices ponderales y tablas de percentiles de los datos antropométricos útiles en una valoración nutricional. Med Clin (Barc) 1988; 91: 223-36.

24. Reid IR, Evans MC, Ames R. Relationships between upper-arm anthropometry and softtissue composition in postmenopausal women. Am J Clin Nutr 1992; 56: 463-6.

25. Chumlea WC, Baumgartner RN. Status of anthropometry and body composition data in elderly subjects. Am J Clin Nutr 1989; 50: 1158-66.

26. Chumlea WC, Guo SS, Kuczmarski RJ, Vellas B. Bioelectrical and anthropometric assessments and reference data in the elderly. J Nutr 1993; 123: 449-53.

27. Bowman BB. Assessment of the nutritional status of the elderly. The American status of the elderly. Am J Clin Nutr 1982; 35:1142-51.

28. Deurenberg P, Weststrate JA, Seidell JC. Body mass index as a measure of body fatness: age and sex specific prediciton formulas. Br J Nutr 65: 105-14, 1991.

29. Menezes TN, Marucci MFN. Perfil dos indicadores de gordura e massa muscular corporal dos idosos de Fortaleza, Ceará, Brasil. Cad Saude Publica 2007 dez; 23(12):2887-95.

30. Marucci MFN, Barbosa AR. Estado nutricional e capacidade física. In: Lebrão ML, Duarte YAO, organizadores. SABE - Saúde, Bem-estar e Envelhecimento. O projeto SABE no Município de São Paulo: uma abordagem inicial. 1. ed. Brasília: Organização Pan-Americana da Saúde; 2003. p. 95- 117.

31. Velázquez-Alva MC, Castillo-Martínez L, Irigoyen-Camacho E, Zepeda-Zepeda MA, Gutiérrez-Robledo LM, Cisneros-Moysen P. Estudio antropométrico en un grupo de hombres y mujeres de la tercera edad en la Ciudad de México. Salud Pública Méx 1996; 38:466-74.

32. Suriah AR, Zalifah MK, Zainorni MJ, Shafawi S, Mimie Suraya S, Zarina N, et al. Anthropometric measurements of the elderly. Malaysian journal of nutrition; 4:55-63.

33. Kuczmarski MF, Kuczmarski RJ, Najjar M. Descriptive anthropometric reference data for older Americans. J Am Diet Assoc 2000; 100:59-66.

34. Santos JL, Albala C, Lera L, García C, Arroyo P, Pérez-Bravo F, et al. Anthropometric measurements in the elderly population of Santiago, Chile. Nutrition 2004; 20:452-7. 
35. Zamboni M, Armellini F, Harris T, Turcato E, Micciolo R, Bergamo-Andreis IA, et al. Effects of age on body fat distribution and cardiovascular risk factors in women. Am J Clin Nutr 1997; 66: 111-5.

36. Rush EC, Plank LD, Laulu MS, Robinson SM. Prediction of percentage body fat from anthropometric measurements: comparison of New Zealand European and Polynesian young women. Am J Clin Nutr 1997; 66: 2-7.
37. Casas YG, Schiller BC, Souza CA, Seals DR. Total and regional composition across age in healthy Hispanic and white women of similar socioeconomic status. Am J Clin Nutr 2001; 73 : 13-8.

38. Miguel Díez J, Grau Carmona T, Izquierdo Alonso JL. Papel de la nutrición en la enfermedad pulmonar obstructiva crónica. Med Clin (Barc) 1998; 110: 307-16.

Recebido: 09/9/2008

Revisado: 14/8/2009

Aprovado: 27/10/2009 
